\title{
Yaron BEN-NAEH, Jews in the Realm of the Sultans. Ottoman Jewish Society in the Seventeenth Century
}

Tübingen, Mohr Siebeck, 2008, 503p.

\section{Annie Benveniste}

\section{(2) OpenEdition}

\section{Journals}

Édition électronique

URL : http://journals.openedition.org/assr/21511

DOI : 10.4000/assr.21511

ISSN : $1777-5825$

Éditeur

Éditions de l'EHESS

Édition imprimée

Date de publication : 31 décembre 2009

Pagination : 75-342

ISBN : 978-2-7132-2218-4

ISSN : 0335-5985

\section{Référence électronique}

Annie Benveniste, "Yaron BEN-NAEH, Jews in the Realm of the Sultans. Ottoman Jewish Society in the Seventeenth Century », Archives de sciences sociales des religions [En ligne], 148 | octobre-décembre 2009, document 148-17, mis en ligne le 27 janvier 2010, consulté le 21 septembre 2020. URL : http:// journals.openedition.org/assr/21511; DOI : https://doi.org/10.4000/assr.21511

Ce document a été généré automatiquement le 21 septembre 2020

(C) Archives de sciences sociales des religions 


\title{
Yaron BEN-NAEH, Jews in the Realm of the Sultans. Ottoman Jewish Society in the Seventeenth Century
}

\author{
Tübingen, Mohr Siebeck, 2008, 503p.
}

\section{Annie Benveniste}

\section{RÉFÉRENCE}

Yaron BEN-NAEH, Jews in the Realm of the Sultans. Ottoman Jewish Society in the Seventeenth Century, Tübingen, Mohr Siebeck, 2008, 503p.

1 L'ouvrage de Yaron Ben-Naeh est une étude de la société juive ottomane du XviI siècle dans ses composantes politique, économique, religieuse et culturelle. La population juive qui vit sous la loi ottomane à cette époque est composée de juifs hellénophones, appelés romaniotes, d'ashkenasim et de juifs expulsés de la péninsule ibérique qui viennent par vagues d'immigration de plus en plus nombreuses sous le règne de BayazidII (1481-1512). Après la conquête des territoires arabes, au début du XVI ${ }^{\mathrm{e}}$ siècle, il faut compter quelques pôles de concentration juive à Bagdad, Alep, Damas et le Caire qui, eux aussi, accueillirent les émigrés ibériques. Bien que ces derniers soient arrivés en nombre et aient manifesté leur supériorité, convaincus de représenter les vraies traditions du judaïsme, on ne peut pas parler d'une identité sépharade qui aurait eu son origine dans la péninsule ibérique et qui se serait maintenue depuis le $\mathrm{xvI}^{\mathrm{e}}$ siècle.

2 L'auteur réfute la thèse d'une identité sépharade en mettant, au contraire, l'accent sur les tensions entre communautés romaniotes et sépharades, d'une part, entre les communautés fondées sur l'origine commune dans la péninsule ibérique, d'autre part, ainsi que sur les mutations que connurent ces dernières depuis leur installation dans les centres urbains de l'Empire ottoman. La mémoire de l'origine - aragonaise, castillane, andalouse... - et l'importance de l'identité spécifique, renforcée par la compétition, font place, à la fin du XVI siècle, à une séphardité inventée, fondée sur la 
mémoire d'un passé fictionnel qui occulte les souvenirs de l'expulsion, de l'errance et des difficultés de l'immigration. La glorification de soi, qui existe à l'arrivée dans l'Empire ottoman, disparait par la suite au profit d'un mouvement d'assimilation favorisé par la stabilité et la sécurité que connaissent les populations juives.

Une seconde mise au point historiographique concerne la qualification de la période analysée, le XVII ${ }^{\mathrm{e}}$ siècle qui fut longtemps considéré comme celui du déclin, mettant l'Empire en position de victime des visées mercantiles des nations européennes. Le siècle commence avec la crise économique et politique, datée des années 1580 , et s'achève avec une autre crise consécutive de la défaite de Vienne en 1683 et inaugurant une série de guerres balkaniques jusqu'à la déposition du sultan Mustapha II. L'auteur rejette le paradigme orientaliste de la dégénération et se propose de revisiter la position sur ce siècle, ce qui l'amène à une autre vision des juifs de l'Empire ottoman. Le $\mathrm{XvII}^{\mathrm{e}}$ siècle doit être considéré comme une période de mutation où l'héritage ibérique des juifs perd certains de ses traits et adopte de nouvelles formes en composant avec les pratiques urbaines du pays.

La première partie de l'ouvrage analyse le contexte démographique et politique de l'Empire, les relations des juifs avec les principales composantes de la société ottomane, les élites, les masses musulmanes et les sujets chrétiens. Les changements essentiels du contexte dans lequel évolue cette société juive ottomane concernent le déclin du pouvoir unique du sultan et l'amorce d'un processus de décentralisation politique par la formation de centres alternatifs du pouvoir dans la capitale et les provinces. La transition d'une société féodale à une société capitaliste s'accompagne du développement d'une bureaucratie professionnelle, de dispositifs de médiation entre gouverneurs et administrés et du renforcement de la charia aux dépens des décrets impériaux. Les raisons qui expliquent le retard économique de l'Empire par rapport à l'Europe sont liées au conservatisme accentué par le pouvoir des guildes et de la loi religieuse. Les importations dont l'État tire profit à travers les taxes limitent tout développement des investissements industriels. La société ottomane, élitiste et monopolistique, est fermée à l'Occident, l'isolationnisme intellectuel ne favorisant pas la pénétration des découvertes scientifiques.

5 La deuxième partie porte sur le kahal - mot hébreu pour «communauté» -, l'analyse de ses institutions, de ses ordonnances et activités ainsi que de ses administrateurs. Les juifs ottomans étaient organisés, selon les principes d'un État pluraliste reconnaissant les minorités, en groupes distincts représentés par les dirigeants de leur kahal. Jusqu'au XIX ${ }^{e}$ siècle, le pouvoir fut oligarchique: l'exemple de la famille Naci montre bien comment des riches ayant des relations avec la cour ottomane et certains de ses représentants exerçaient un véritable contrôle sur les communautés et dictaient leur politique. L'auteur concentre son étude sur la judaïcité urbaine en privilégiant les communautés d'Istanbul, de Salonique et d'Izmir qui comptent parmi les principales concentrations juives et d'où provient également la plus grande partie des sources écrites disponibles. Les autres centres importants, sur le plan économique et pour leur population juive, sont situés dans les provinces arabes et les sources sont rares, sauf en ce qui concerne Jérusalem. L'importance des relations intercommunautaires, de la diffusion des réseaux culturels, religieux ou d'assistance est une occasion de confronter les données sur les centres principaux et leurs périphéries comme Edirne ou Safed et de montrer comment la compétition entre les villes s'exerce aussi bien dans le domaine 
religieux que dans le domaine économique, faisant passer le leadership de Salonique à Jérusalem.

6 Une dernière partie s'attache à l'analyse économique, sociale et culturelle de la société juive ottomane. Les juifs participent à tous les niveaux des échanges commerciaux urbains dans les caravansérails, les ports ou les rues comme boutiquiers, marchands itinérants ou colporteurs. On les trouve également engagés dans le commerce des esclaves, les services bancaires et dans la collecte des impôts et taxes diverses. Mais leur implication dans la vie économique est relativement diversifiée puisqu'ils sont aussi spécialisés dans l'industrie du textile et dans la joaillerie. Cette diversité dans l'activité économique se traduit, sur le plan social, par une inégalité des positions hiérarchiques, l'élite financière ou commerçante étant relativement réduite par rapport à une masse de petits artisans ou journaliers qui ne pouvaient survivre que grâce à l'assistance de la communauté. La judaïcité ottomane est donc diversifiée mais reste globalement une société religieuse traditionnelle. L'héritage du judaïsme est essentiel dans la définition de l'identité individuelle et collective ainsi que du mode de vie dans son ensemble. L'identité religieuse dicte à la fois les pratiques de la vie quotidienne, le statut légal, les relations sociales et familiales, l'éducation et le type de résidence. Mais la société traditionnelle est traversée par des tensions entre les masses pieuses, d'une part, des individus transgressifs et des mouvements piétiste ou kabbaliste, d'autre part. Les modes d'expression de l'identité juive ne sont pas univoques, entre l'adoption d'un costume spécifique et l'identification aux classes supérieures. La loi ottomane exige la séparation des groupes en fonction de la classe et de la religion mais la dynamique de la vie urbaine commande un certain nombre d'échanges, qu'il s'agisse de cohabitations résidentielles, d'interactions dans les marchés ou dans les guildes, des temps partagés dans les cafés ou d'autres espaces récréatifs. Les membres de l'élite économique juive, qui fournissent en produits de luxe ou en services financiers les membres de la cour, ont accès à cette dernière et aux maisons des leaders politiques et militaires. Nombre de marchands, d'intermédiaires, d'interprètes sont en relation avec les commerçants et les voyageurs européens et jouent un rôle important dans l'importation de produits et modèles culturels occidentaux.

7 L'auteur justifie ainsi la notion de société juive ottomane en montrant qu'à partir du milieu du XVI ${ }^{e}$ siècle, la loi ottomane cessa d'être étrangère et que l'assimilation à cette culture joua un rôle majeur dans la vie quotidienne. On assiste à une interpénétration de la société juive et des institutions urbaines. Désormais, la culture juive peut être interprétée comme un mixte de deux héritages, ibérique et ottoman, le judaïsme autochtone, romaniote ou arabe, étant réduit à l'état de survivance.

Ce livre, traduit de l'hébreu et qui présente une étude de la société juive urbaine du $\mathrm{xVII}^{\mathrm{e}}$ siècle, comble une lacune dans l'historiographie des juifs ottomans. Elle est menée à partir de sources diverses, hébraïques et autres, publiques et privées. Elle évite le principe de la monographie pour décrire des communautés de moyenne et large importance, non dans leur spécificité mais dans leurs relations les unes aux autres et avec le contexte global, en mettant l'accent sur les tensions et les contradictions internes, comme sur les mutations. 